\title{
GNSS and its impact on position estimates
}

\author{
Chiranjeevi G. Vivek ${ }^{1,2}$, T. S. Shrungeshwara ${ }^{1}$ and Sridevi Jade ${ }^{1,2, *}$ \\ ${ }^{1}$ CSIR-Fourth Paradigm Institute (CSIR-4PI), Wind Tunnel Road, Bengaluru 560 037, India \\ ${ }^{2}$ Academy of Scientific and Innovative Research (AcSIR), Ghaziabad 201 002, India
}

This study evaluates the impact of multi-GNSS (Global Navigation Satellite System) signals on the estimation of precise position with millimetre accuracy. Compared to standalone satellite systems like the Global Positioning System (GPS), multi-GNSS improves start-up time, performance, satellite visibility, accuracy, spatial geometry and reliability. However, on the flip side it increases the noise, signal interference, hardware complexity of the receiver, intersystem interference and computation complexity which may degrade its performance. Though GNSS is similar at fundamental levels, differences exist in signal structures, reference frames and timing standards. Compatibility and interoperability between the different constellations of the highest order is required to achieve the best results. At present, GPS and Glonass navigation systems are fully functional with global coverage and comparable precision. Glonass satellite constellation, signal structure is slightly different when compared to GPS, whereas major differences exist in the reference frame and epoch time. Combined GPS-Glonass solution significantly improves the accuracy in navigation applications with increased satellite signal observations and spatial distribution of visible satellites. For precise geodetic studies using static post-processing, combined solution may degrade the accuracy, if these differences are not handled carefully. Currently for geodetic studies, only GPS observations are majorly used worldwide. For the first time, daily precise position is estimated for continuous GNSS stations located in India using static postprocessing with standalone GPS, Glonass as well as combined GPS-Glonass to study the impact of multiGNSS signals for geodetic studies.

Keywords: Glonass, GNSS signals, GPS, multi-GNSS data analysis, position.

GLOBAL Navigation Satellite System (GNSS) consists of global and regional satellite navigation systems along with their control and user segments. The global satellite navigation systems are Global Positioning System (GPS), Glonass, Galileo, BeiDou, of which GPS operated by USA and Glonass operated by Russia are currently fully functional. European Galileo and Chinese BeiDou are in different phases of satellite and ground infrastructure development. Regional satellite navigation systems such

*For correspondence. (e-mail: sridevi@ csir4pi.in) as Japanese QZSS (Quasi-Zenith Satellite System) and Indian Regional Navigation Satellite System NavIC (Navigation using Indian Constellation) improve the navigation position estimates over these countries. GNSS is dynamically changing with several new satellites being launched by different countries worldwide.

Satellite navigation is allocated to the microwave frequencies of $\mathrm{L}(1-2 \mathrm{GHz}), \mathrm{S}(2-4 \mathrm{GHz})$ and $\mathrm{C}(4-8 \mathrm{GHz})$ bands. Most of the GNSS signals use code division multiple access (CDMA), except legacy Glonass signals which use the frequency division multiple access (FDMA) technique. GNSS signals contain ranging codes and navigation data to provide positioning, navigation and timing (PNT) services. GNSS signals are pure sinusoidal signals with binary codes (coarse acquisition (C/A) code, precision (P) code) modulated on carrier signals. Binary phase shift keying (BPSK) modulation is most widely used and data is conveyed by changing the phase of the carrier signal depending on the code signal. Nextgeneration GNSS uses binary offset carrier (BOC) modulation in which the BPSK signal is multiplied with square-wave sub-carrier to reduce the multipath effect, signal interference and is currently implemented in GPS, NavIC and Galileo. GNSS strongly relies on measuring the time of arrival of radio signals and hence space, control and user segments are time-synchronized. GNSS architecture and signal structure is explained in detail in the Supplementary material.

Performance of multi-GNSS depends on interoperability at system and signal level, radio-frequency compatibility and spectral separation. GPS and Galileo (except $\mathrm{E}_{6}$ ) signals are interoperable and compatible, whereas Glonass and BeiDou groups are still working towards achieving compatibility and interoperability. Regional systems QZSS and NavIC are fully compatible and interoperable with GPS and hence improve the positional accuracy in the respective regions. Even though GNSS are similar at fundamental levels, differences exist in reference frames, timing standards and signal structures which need to be addressed to achieve the best result.

GNSS user community can be broadly classified based on the applications and accuracy requirements such as mobile (single-point positioning), navigation (differential positioning) and geodetic studies (static relative positioning). For mobile users, multi-GNSS improves the accuracy and satellite visibility, especially in urban and forest areas. For navigation application, the required accuracy is 
RESEARCH ARTICLES

Table 1. GPS and Glonass observation equations

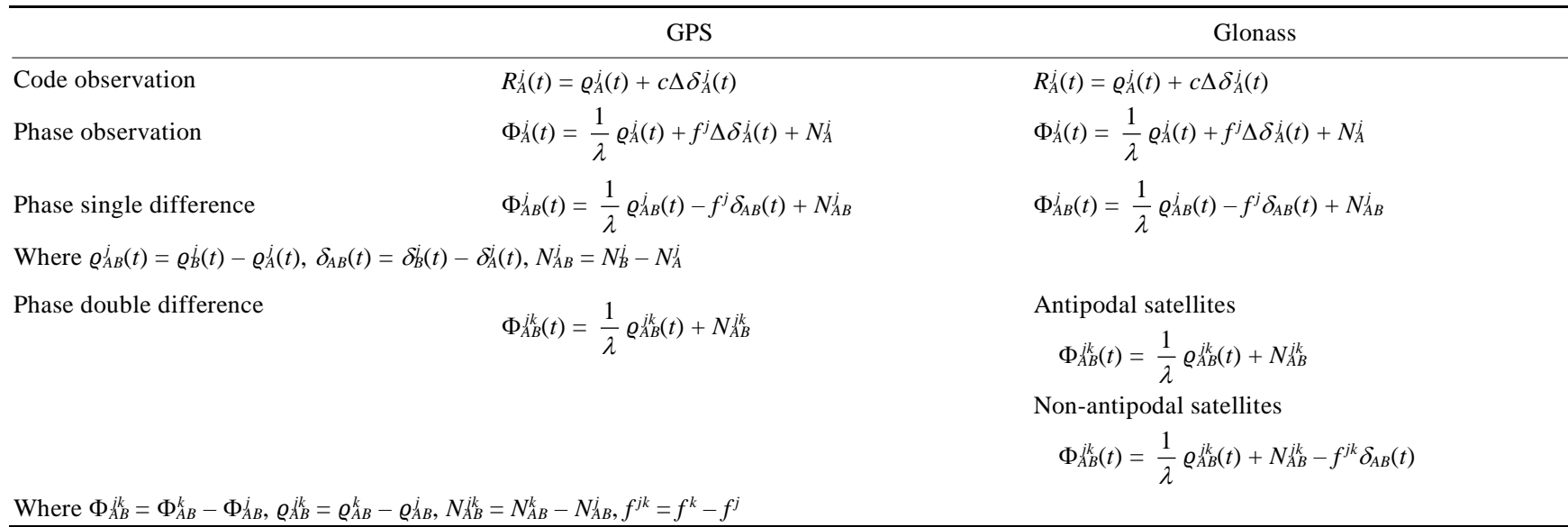

$j, k$ are satellites; $A, B$ are receivers; $R_{A}^{j}(t)$ is the code pseudorange between satellite $(j)$ and receiver $(A)$ at an epoch $t ; \varrho_{A}^{j}(t)$ is the geometric distance between satellite $(j)$ and receiver $(A) ; c$ is the velocity of light, $\Delta \delta_{A}^{j}(t)$ is the combined satellite $(j)$ and receiver $(A)$ clock bias; $\Phi_{A}^{j}(t)$ is the measured carrier phase; $\lambda$ is the wavelength; $N_{A}^{j}$ is the integer ambiguity; $f^{j}, f^{k}$ are the frequency of satellites $j$ and $k$ respectively.

in the order of few metres, and multi-GNSS reduces the convergence time and increases positional accuracy. Since tracking more satellites increases the power consumption and hardware design complexity, suitable trade-off with the requirements is needed to design costeffective receivers based on the applications. For scientific research, i.e. geodetic studies where millimetre $(\mathrm{mm})$ level accuracy is required, GNSS data is post-processed in static mode to determine the precise position. For combining different constellations, it is required to have a unique reference system, satellite ephemerides and a unique timescale for all the observations for all satellites and receiver positions. Here, we report a pilot study to assess the impact of multi-GNSS on multi-year position estimates specific to the Indian subcontinent.

\section{Multi-GNSS data analysis for geodetic studies}

GPS and Glonass measurement types are C/A-code on $\mathrm{L}_{1}$, P-code on $\mathrm{L}_{1}, \mathrm{~L}_{2}$ and carrier phase on $\mathrm{L}_{1}, \mathrm{~L}_{2}$, which represent the observables for post-processing. For geodetic applications, code and phase data of the satellites are post-processed in static mode using precise satellite orbits, troposphere, ionosphere models, etc. Pseudo range $\left(\varrho_{A}^{\dot{L}}(t)\right)$ is the distance between satellite $(j)$ and receiver $(A)$ at a specific time $(t)$. The receiver generates a copy of the code generated by the satellite for signal modulation and shifts it until maximum correlation with the received signal occurs and the code pseudo-range equation $\left(R_{A}^{\dot{i}}(t)\right)$ is formulated. For carrier phase signal, the receiver records the instant phase difference $\left(\Phi_{A}^{\dot{A}}(t)\right)$ between the phase signal generated by satellite $(j)$ and receiver $(A)$ based on which the phase observation equation is formulated. The observation equations for code and phase are used to form single, double and triple differences using linear combinations to eliminate/reduce existing biases and errors induced in the signal during its transit from the satellite to the receiver.

\section{Stand-alone GPS and Glonass static post processing}

Table 1 gives the code and phase observables for standalone GPS and Glonass. Single difference $\left(\Phi_{A B}^{\dot{j}}(t)\right)$ of the phase observables between two receivers $(A, B)$ and a single satellite $(j)$ eliminates the satellite clock bias term $\left(\Delta \delta^{j}(t)\right)$ for both the GPS and Glonass constellations. Double difference solution $\left(\Phi_{A B}^{j k}(t)\right)$ between two stations $(A, B)$ and two satellites $(j, k)$ eliminates receiver clock bias $\left(\delta_{A B}(t)\right)$ for GPS. If $j, k$ are antipodal Glonass satellites, then both satellites transmit in the same frequency removing the receiver clock bias term and $\left(\Phi_{A B}^{k}(t)\right)$ is same as for GPS. If $j, k$ are non-antipodal satellites, $f^{j} \neq f^{k}$ and hence $f^{j k} \delta_{A B}(t)$ (single difference bias term) remains in double difference equation, unlike in GPS. Hence, receiver clock errors do not cancel and propagate as additional ambiguities for both standalone Glonass and combined GPS/Glonass data post-processing ${ }^{1}$.

\section{Combined GPS/Glonass data static post processing}

In order to combine GPS and Glonass data for static postprocessing, a unique reference system, combined satellite ephemerides and a unique timescale are needed for all the observations ${ }^{1}$. In addition, difference between frequencies of GPS/Glonass satellite pairs needs to be considered, which increases the single difference bias term in the double difference phase observable.

Transformation parameters for Glonass reference frame (PZ-90). Parametry Zemli 1990 (PZ-90) is a Glonass reference frame consisting of fundamental geodetic 
constants, earth ellipsoid, gravity field parameters, geocentric coordinate system and transformation parameters. PZ-90.11 is the latest geocentric coordinate system ${ }^{2}$ used for geodetic support of orbital missions and navigation from 15 January 2014. Transformation parameters between PZ-90.11 and World Geodetic System (WGS)-84 are used to combine Glonass and GPS broadcast ephemerides.

Combined GPS/Glonass orbits. To process GPS and Glonass observations in the combined mode, positions of GPS, Glonass satellites and all receivers must refer to a unique reference system ${ }^{1}$. Broadcast ephemerides are used as apriori orbit information. Different reference systems for GPS, Glonass require generation of the combined set of GPS/Glonass orbits in WGS-84 and GPS time (Figure 1). Epochs of the Glonass ephemerides are approximately corrected to GPS time (applying the leap seconds between Coordinated Universal Time (UTC) and GPS time) and satellite positions are interpolated to the epochs of the GPS positions. Interpolated positions are transformed to WGS-84 and the result is saved in SP3 file $^{3}$, which are available with a time delay of $12-18$ days.

System time differences. System time difference can be estimated based on three observation types, i.e. observations only to GPS satellites, observations only to Glonass satellites and observations to both the GPS and Glonass satellites ${ }^{1}$. Three assumptions used for processing are: (i) epochs of observations and ephemerides must refer to a unique timescale (UTC or GPS); (ii) receiver clock needs to be synchronized to a unique timescale, and (iii) GPS, Glonass observations of the receiver need to be performed simultaneously or with known delay. Here an epoch refers to the time tag for a specific set of observations, clocks or ephemerides.

In the observations only to GPS or Glonass satellites, the first two assumptions hold good and estimation of

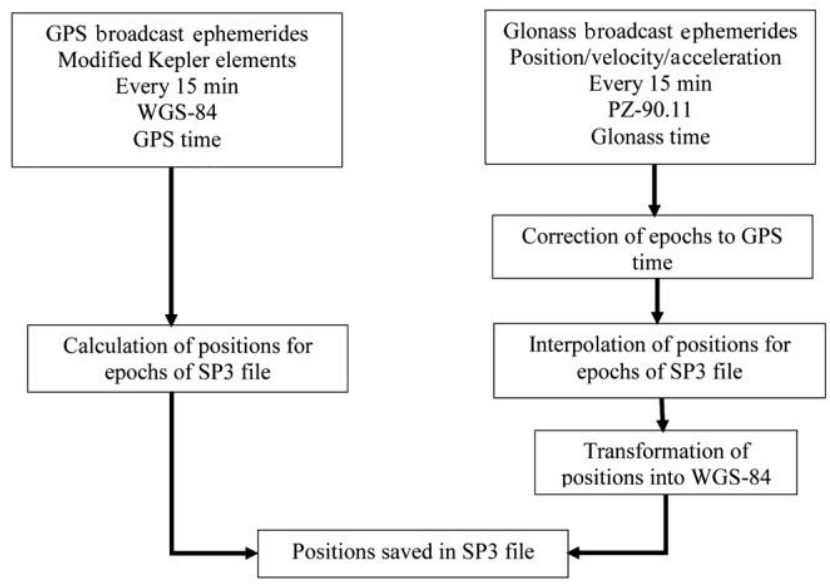

Figure 1. Combined GPS/Glonass orbit generation. time difference between GPS and Glonass is not required. In case of both GPS and Glonass observations, if the receiver clock is assumed to synchronize with GPS time, epochs of Glonass observations refer to GPS time, but the ephemerides are still given in Glonass time and the first assumption does not hold. Hence epochs of the Glonass ephemerides need to be corrected for a known number of leap seconds ${ }^{1}$.

Combined GPS-Glonass observables. Combined code pseudo range observation equation is

$$
\begin{aligned}
& R_{A}^{\dot{A}}(t)=\varrho_{A}^{\dot{A}}(t)+c\left(\Delta \delta_{\dot{A}}^{\dot{y}}(t)+\Delta t^{s}\right) \\
& \Delta t^{s}=t_{\mathrm{GPS}}-t_{\mathrm{GLONASS}}-n,
\end{aligned}
$$

where $c=3 \times 10^{8} \mathrm{~m} / \mathrm{s}$ and $n$ is the leap second.

In combined GPS/Glonass data-processing, the code pseudo range equation has an extra term $\Delta t^{s}$. If GPS time is considered, then $\Delta t^{s}=0$ for GPS observations and $\Delta t^{s} \neq 0$ for Glonass observations.

Phase observation equation of combined GPS and Glonass is

$$
\Phi_{A}^{\dot{j}}(t)=\frac{1}{\lambda} \varrho_{A}^{\dot{A}}(t)+f^{j} \Delta \delta_{A}^{\dot{j}}(t)+N_{A}^{\dot{j}}+c \Delta t^{s},
$$

where $\lambda$ is the signal wavelength and $N_{A}^{j}$ is the integer ambiguity.

Here, $\Delta t^{s}$ is a combination of system-dependent term $\left(\Delta t^{v}\right)$ and receiver-dependent term $\left(\Delta t_{r}\right)$

$$
\Delta t^{s}=\Delta t^{v}+\Delta t_{r},
$$

where $\Delta t^{v}=\tau_{c}+\tau_{u}+\tau_{g}, \quad \tau_{c}=t_{\mathrm{UTC}(\mathrm{SU})}-t_{\mathrm{Glonass}} ; \tau_{u}=t_{\mathrm{UTC}}-$ $t_{\mathrm{UTC}(\mathrm{SU})} ; \tau_{g}=t_{\mathrm{GPS}}-t_{\mathrm{UTC}}$

Here SU stands for Soviet Union, and $\tau_{c}, \tau_{u}$ and $\tau_{g}$ are updated and provided by the Bureau International Des Poids Et Mesures (BIPM), France.

Hence, the phase observable for Glonass satellite $j$ and GPS satellite $k$ is

$$
\begin{aligned}
& \Phi_{A}^{\dot{A}}(t)=\frac{1}{\lambda} \varrho_{A}^{\dot{A}}(t)+f^{j} \Delta \delta_{A}^{\dot{A}}(t)+N_{A}^{j}+c \Delta t^{v}+c \Delta t_{r}, \\
& \Phi_{A}^{k}(t)=\frac{1}{\lambda} \varrho_{A}^{k}(t)+f^{k} \Delta \delta_{A}^{k}(t)+N_{A}^{k} .
\end{aligned}
$$

Single difference phase equation is

$$
\begin{aligned}
& \Phi_{A B}^{\dot{j}}(t)=\frac{1}{\lambda} \varrho_{A B}^{\dot{A}}(t)-f^{j} \delta_{A B}(t)+N_{A B}^{\dot{j}}+c \Delta t_{r A B}, \\
& \Phi_{A B}^{k}(t)=\frac{1}{\lambda} \varrho_{A B}^{k}(t)-f^{k} \delta_{A B}(t)+N_{A B}^{k},
\end{aligned}
$$


where $\Delta t_{r A B}=\Delta t_{r A}-\Delta t_{r B}$, and $\Delta t^{\nu}$ is removed in single difference solution.

Double difference phase observable of Glonass/GPS satellite pair is

$$
\Phi_{A B}^{i k}(t)=\frac{1}{\lambda} \varrho_{A B}^{j k}(t)+N_{A B}^{j k}-f^{j k} \delta_{A B}(t)+c \Delta t_{r A B} .
$$

Receiver-dependent term $\left(\Delta t_{r A B}\right)$ is not eliminated in double difference observable and this term increase ambiguities in addition to $f^{j k} \delta_{A B}(t)$. In the above equation, relative difference between receivers $(A, B)$ results in ambiguity which exists only in the case of GPS/Glonass satellite pair. To solve combined GPS/Glonass observation ambiguities, three double difference types are used ${ }^{1}$.

Type 1: GPS-GPS/Glonass-Glonass difference with the same frequencies: If two Glonass satellites with identical carrier frequencies are visible at different observation epochs, then the single difference bias term is zero and ambiguity resolution is independent of initialization.

Type 2: Glonass-Glonass difference for different frequencies: This ambiguity can be resolved if the bias term does not affect the integer nature of the ambiguities. The ambiguity resolution algorithm reduces the size of the bias term after each successful iteration.

Type 3: GPS-Glonass difference: The bias term is very large due to the wavelength difference and a different ambiguity resolution algorithm is used. First, all ambiguities referring to two GPS or Glonass (same frequencies) satellites are resolved followed by non-antipodal satellite ambiguities and then GPS and Glonass satellite ambiguities, provided there is no significant receiver bias between the two systems.

\section{Processing of cGNSS data of Indian subcontinent}

GNSS data of five continuous GNSS (cGNSS) stations located in India along with seven multi-GNSS global IGS (International GNSS Service) sites (Figure 2) during 2016-2019 were analysed using Bernese software version 5.2 (ref. 4), to estimate the daily positions from standalone GPS (GPS), Glonass (GLO) and combined (GGL) GPS/Glonass processing. Products provided by the Centre for Orbit Determination in Europe (CODE) for the final earth rotation parameters (ERP), precise GPS and Glonass satellite orbits, $30 \mathrm{sec}$ final clock products from the final IGS solution, monthly differential code bias (DCB) estimates, antenna phase centre, satellite and ionosphere information were used in the analysis ${ }^{5}$. The cGNSS data were converted to intermediate format (tgd) using runpkr00 version 5.40, and then converted to RINEX (receiver independent exchange) format version 2.11 using TEQC (Translation, Editing and Quality Check) software version 2018 oct 15 .

BPE (Bernese processing engine) designed for automatic processing of cGNSS networks was used for the analysis in which tasks were defined using PCFs (process control files). PPP_BAS and RNX2SNX PCFs allow combined GPS/Glonass processing, whereas full ambiguity resolution with GPS/Glonass is supported only in RNX2SNX PCF. PPP_BAS.PCF (precise point positioning) generates apriori information for double difference analysis using RNX2SNX PCF. The first step is to form single difference observation files by selecting baselines between stations using 'OBS-MAX' (maximum observations) strategy, then fixing $L_{1}, L_{2}$ ambiguity parameters to determine the final station coordinates ${ }^{4}$. In double difference analysis, station coordinates and troposphere parameters were estimated and stored in Bernese and SINEX (solution independent exchange) formats. Sampling interval of $30 \mathrm{sec}$ and elevation cut-off angle of $10^{\circ}$ were used in the analysis.

For GPS and Glonass data, ambiguities were resolved using sigma strategy: (i) directly on $L_{1}$ and $L_{2}$ for baseline lengths of less than $20 \mathrm{~km}$; (ii) phase-based wide-lane ambiguities for baseline length of $20-200 \mathrm{~km}$, and (iii) quasi ionosphere free (QIF) strategy for baseline length of $200-2000 \mathrm{~km}$. QIF ambiguity resolution strategy is very sensitive to formal errors of the ambiguity parameters and needs measurements with $30 \mathrm{sec}$ sampling. For baseline lengths of $2000-6000 \mathrm{~km}$, wide-lane and narrow-lane ambiguities were resolved using Melbourne-Wubbena (MW) linear combination for GPS data. Single difference bias term (as detailed in section Stand-alone GPS and Glonass static post processing) due to inter-frequency code biases caused by the FDMA technology of Glonass is not explicitly handled in the software. Zenith tropospheric

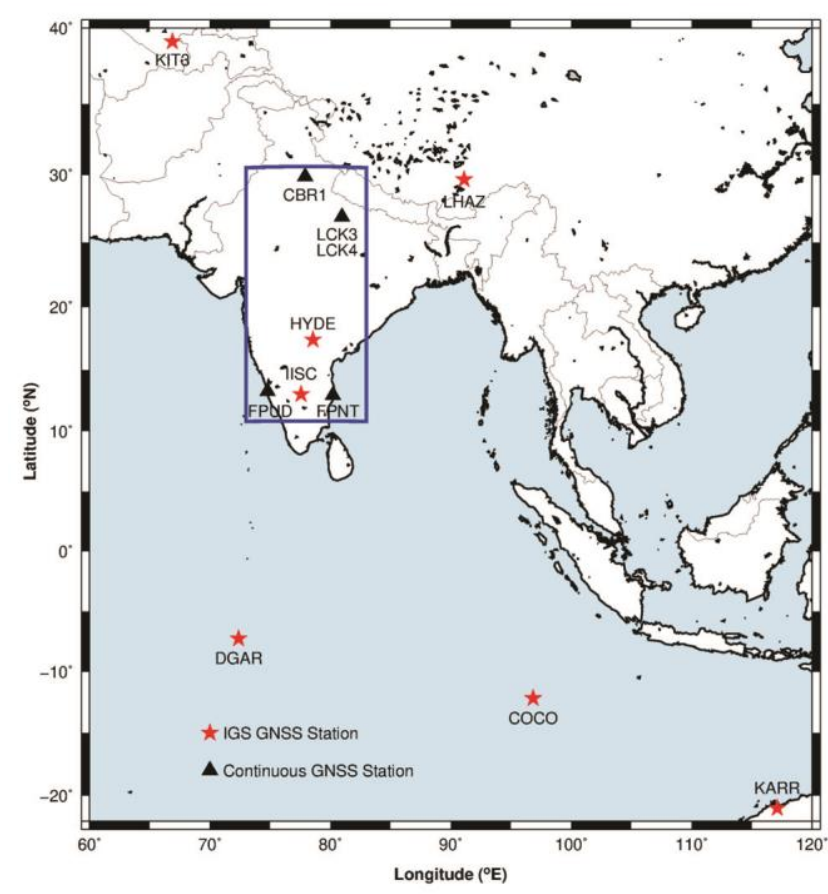

Figure 2. cGNSS sites of India (boxed) along with the IGS stations with GNSS data. 
delay for each station was estimated by incorporating a piecewise linear model with stochastic constraints and then corrected for the signal delay due to troposphere. Global pressure temperature (GPT) model was used with dry and wet global mapping function (GMF). Horizontal tropospheric gradients have been represented in a piecewise linear way and Chenher gradient estimation model was used. Daily precise positions and velocities (GPS, GLO and GGL) of the cGNSS sites were determined by constraining the seven IGS stations in International Terrestrial Reference Frame ITRF2014 (ref. 6).

\section{Results and discussion}

Figure 3 is a plot of daily north, east and up components of the coordinate time series for standalone and combined GPS-Glonass for the 60 days. Daily position estimates indicate high scatter for standalone Glonass when compared to GPS and GGL data. This is due to errors associated with the receiver clock bias term $\left(\delta_{A B}(t)\right)$ in double difference solution of Glonass data (as detailed in section Stand-alone GPS and Glonass static post processing; Table 1). This introduces additional ambiguity terms to be resolved for non-antipodal Glonass satellites. In addition, daily position estimates indicate high scatter for the east component compared to the north component for GPS,
GLO and GGL. Since IGS stations equipped with multiGNSS receivers are limited in the Indian subcontinent region (Figure 2) with only two stations located within India along $\mathrm{N}-\mathrm{S}$, the east component could not be well constrained in the solution in addition to contribution from unresolved ambiguities. Time series of up component indicates 2-3 times high scatter compared to the north and east components as satellite positioning traditionally estimates the horizontal positions 2-3 times more precisely than vertical positions ${ }^{7}$. Vertical accuracies are low as the satellite constellation is inconsistent for surface-based receivers and also due to increased errors in code/phase solutions. Combined GPS-Glonass solution does not indicate any significant improvement compared to standalone GPS solution as the receiver-dependent term $\left(\Delta t_{\mathrm{r} A B}\right)$ is not eliminated in double difference observable and it contributes to additional ambiguities (as detailed in section Combined GPS-Glonass observables). Wang et al. ${ }^{8}$ identified crucial issues to be resolved in using standard double difference procedure for GLO and GGL data due to multiple frequencies of Glonass signals. They suggested several mathematical, stochastic models and ambiguity resolution strategies for analysing standalone Glonass and combined GPS-Glonass data. Further, modelling of $\mathrm{L}_{3}$ phase observable of Glonass causes a mean difference of up to $1 \mathrm{~cm}$, which gets partially offset
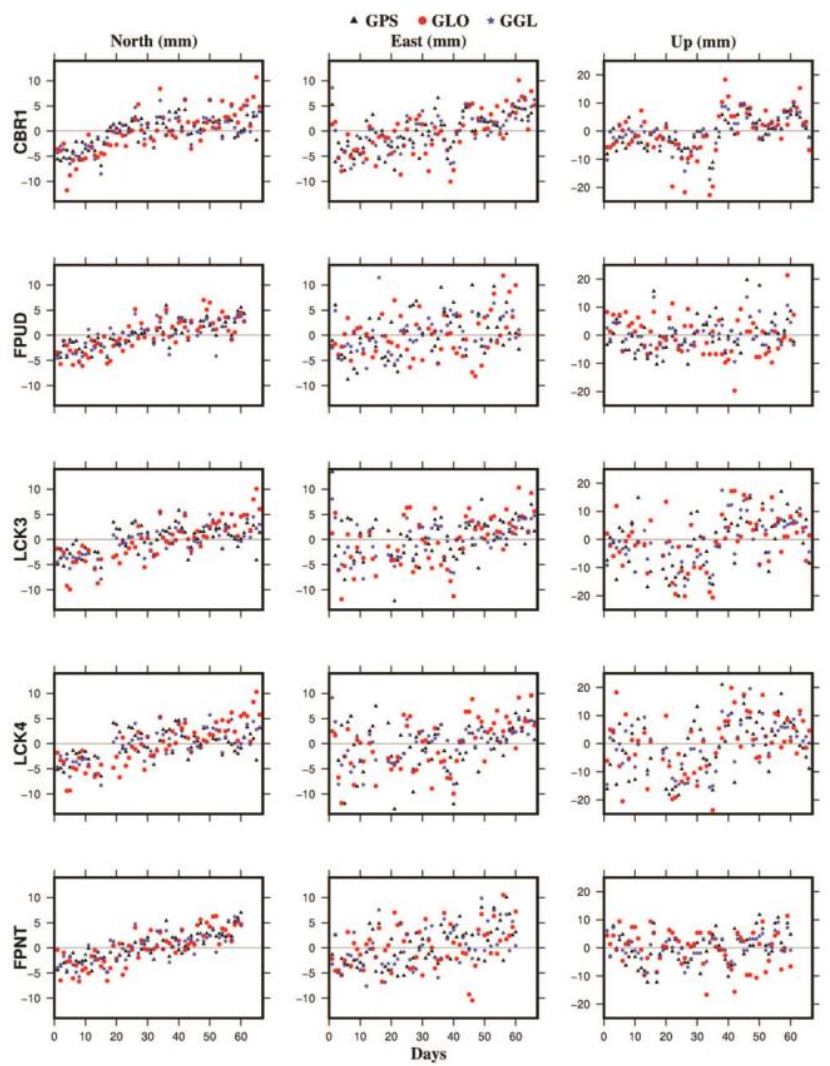

Figure 3. Daily position time series in north, east, and up components using GPS, GLO and GGL of Indian cGNSS sites.
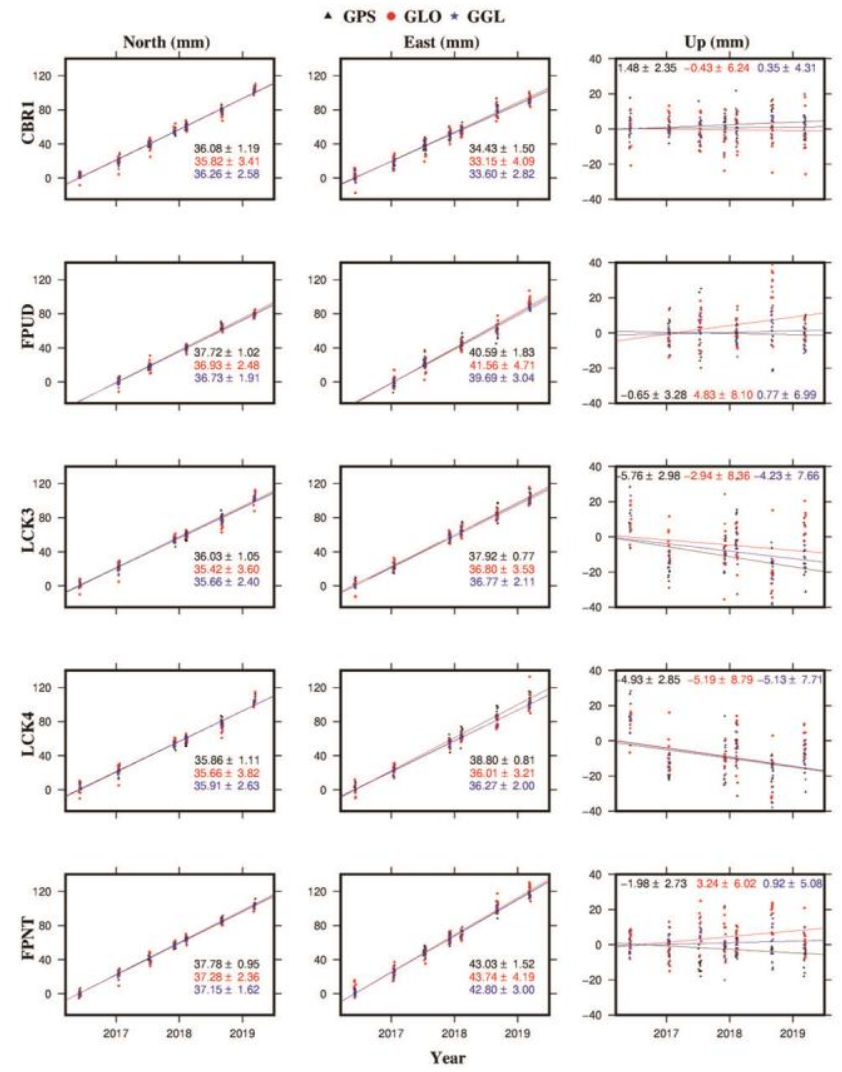

Figure 4. Multi-year time series of north, east, and up components for GPS, GLO and GGL solutions of Indian cGNSS sites. 

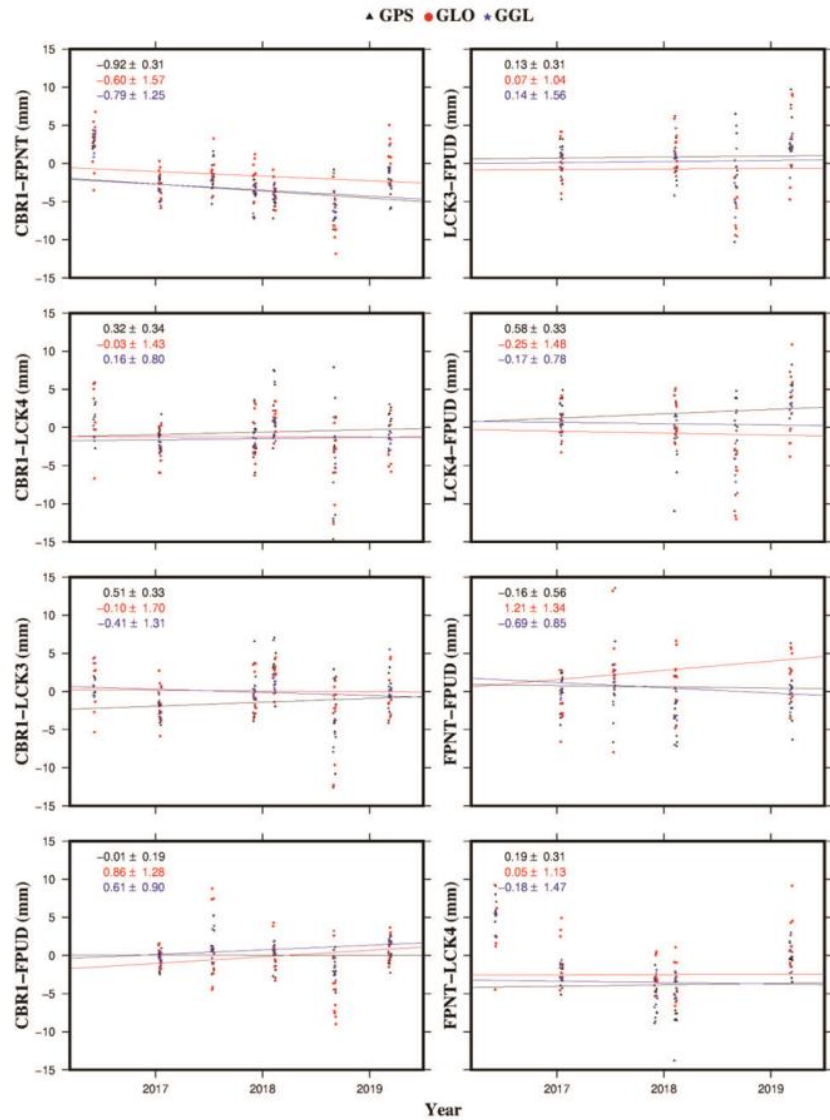

Figure 5. Time series of baseline lengths of about 500-2000 km for GPS, GLO and GGL multi-year solutions.

in the GGL solution ${ }^{9}$. In addition, errors in Glonass orbits cause an error of $4 \mathrm{~mm}$ for baseline lengths of $2000 \mathrm{~km}$ and above ${ }^{10}$.

Figures 4 and 5 are plots of multi-year time series of north, east and up components of daily positions and baselines lengths respectively, for GPS, GLO and GGL solutions. Scatter is high for the GLO solution compared to GPS and GGL solutions. The north component of velocity for all the sites differs by $\pm 1 \mathrm{~mm}$, well within the nrms (normalized root mean square) error value for GPS, GLO and GGL solutions. Large difference (1-3 $\mathrm{mm})$ is observed for the east component of velocity for the three solutions, which is due to insufficient/skewed spatial spread of IGS stations with multi-GNSS data used for defining the reference frame and ambiguity resolution algorithms. Height rates are statistically insignificant with high scatter, i.e. nrms of about $3-8 \mathrm{~mm}$ in daily solutions. A previous study for regional EUREF permanent network (EPN) indicated about 1-2 mm difference between GPS-only and GPS + Glonass coordinates, which is mainly due to reference frame differences of the regional networks ${ }^{11}$, and the GGL solution does not improve precision of position estimates. Several authors showed that additional observations of Glonass do not contribute to the precision in spite of better observation geometry, which is offset by poor accuracy of Glonass orbits and clock corrections ${ }^{12-14}$. Multi-year (2.5 years) of daily solutions indicates that GGL solutions show little improvement when compared to GPS-only solutions ${ }^{15}$.

\section{Conclusion}

Static post-processing of combined GPS and Glonass observables requires (a) common satellite ephemerides files in GPS timescale, (b) an additional parameter to account for the time difference in code and phase observation equations of combined GPS/Glonass observations, and (c) additional single difference ambiguity term at the double difference level for Glonass phase observation equations of satellite specific frequencies, which affects the cycle slip detection and ambiguity resolution. Combined GNSS solution for geodetic studies is complex and may result in noisy position estimates if the above aspects are not resolved correctly.

This pilot study indicates that multi-GNSS does not significantly improve the positional accuracy, but it eliminates the dependency on a particular satellite system in the long term. Position time series of multi-GNSS is much more stable than single GNSS with errors. For the Indian subcontinent, GPS solution gives precise estimates of position and rates compared to Glonass and combined 
GPS-Glonass solutions. This is due to poorly resolved additional ambiguity terms in GLO and GGL solutions for long baselines, and also the spatial spread of IGS sites equipped with multi-GNSS receivers is not sufficient enough to enable a well-constrained robust reference frame for GGL solutions. Comprehensive analysis of minimum of three years of continuous GNSS data to determine position and velocity estimates would give a true picture of the noise levels and accuracy involved in standalone and combined GPS/Glonass analysis for the Indian subcontinent.

Satellite navigation is undergoing dramatic changes with the development of multi-GNSS constellations with an estimated $\sim 140$ GNSS satellites in space by the end of 2020. The future of satellite navigation is in using combined GNSS solutions to significantly improve the performance, satellite visibility, dilution of precision, accuracy, spatial geometry, redundancy and reliability.

1. Habrich, H., Geodetic applications of the Global Navigation Satellite System (GLONASS) and of GLONASS/GPS combinations, $\mathrm{PhD}$ thesis, University of Bern, Switzerland, 1999.

2. Parametry Zemli 1990 (PZ-90.11) - Reference document, Military Topographic Department of the General Staff of Armed Forces of the Russian Federation, Moscow, 2014.

3. Remondi, B. W., Extending the national geodetic survey standard orbit formats. Technical Report Nos 133, NGS 46, NOAA, Rockville, Maryland, USA, 1989.

4. Dach, R. et al. (eds), Bernese GNSS Software Version 5.2, User Manual, Bern Open Publishing, Astronomical Institute, University of Bern, Switzerland, 2015.

5. Dach, R. et al., CODE Final Product Series for the IGS, Astronomical Institute, University of Bern, Switzerland, 2018.

6. Altamimi, Z., Rebischung, P., Metivier, L. and Collilieux, X., ITRF2014: a new release of the International Terrestrial Reference
Frame modeling nonlinear station motions. J. Geophys. Res., Solid Earth, 2016, 121; doi:10.1002/2016JB013098.

7. Hofmann-Wellenhof, B., Lichtenegger, H. and Collins, J., Global Positioning System Theory and Practice, Third revised edition, Springer-Verlag GmbH, Wien, Austria, 1994; doi:10.1007/978-37091-3311-8.

8. Wang, J., Rizos, C., Stewart, M. and Leick, A., GPS and GLONASS integration: modelling and ambiguity resolution issues. GPS Solut., 2001, 5, 55-64.

9. Dach, R. et al., Improved antenna phase centre models for GLONASS. GPS Solut., 2011, 15, 49-65.

10. Beutler, G. et al., Bernese 5 GPS Software User Manual, Astronomical Institute, University of Bern, Switzerland, 2007.

11. Bruyninx, C., Comparing GPS-only with GPS+GLONASS positioning in a regional permanent GNSS network. GPS Solut., 2007, 11(2), 97-106.

12. Habrich, H., Evaluation of analysis options for GLONASS observations in regional GNSS networks, Geodetic Reference Frames. In Proceedings of the International Association of Geodesy Symposia, 2009, vol. 134, pp. 121-129.

13. Dach, R. et al., GNSS processing at CODE: status report. J. Geod., 2009, 83, 353-365.

14. Dach, R. et al., Improved antenna phase center models for GLONASS. GPS Solut., 2011, 15, 49-65.

15. Nardo, A., Huisman, L. and Teunissen, P. J. G., GPS + GLONASS CORS processing: the Asian-Pacific APREF case. In Earth on the Edge: Science for a Sustainable Planet (eds Rizos, C. and Willis, P.), International Association of Geodesy Symposia, 2014, vol. 139, pp. 239-246.

ACKNOWLEDGEMENTS. This is CSIR-4PI ARiEES contribution. This research work is part of doctoral work of first author and he acknowledges the encouragement of doctoral advisory committee members and co-supervisor. We acknowledge the support of Head, CSIR-4PI for the GNSS programme.

Received 8 August 2019; revised accepted 28 July 2020

doi: $10.18520 / \mathrm{cs} / \mathrm{v} 119 / \mathrm{i} 9 / 1503-1509$ 